\title{
Species Richness and Abundance of Major Insect Pollinators on Aonla, (Phyllanthus emblica L.)
}

\author{
Anoosha Vadde $^{1 *}$, Sumit Saini ${ }^{2}$, H.D. Kaushik ${ }^{1}$ and Sunita Yadav ${ }^{1}$ \\ ${ }^{1}$ Department of Entomology, CCS Haryana Agricultural University, \\ Hisar, Haryana, 125004, India \\ ${ }^{2} C I B \&$ RC, Faridabad, Haryana, India \\ *Corresponding author
}

\section{A B S T R A C T}

Aonla blossoms attracted total twelve insect species belonged to 5 orders, 7 families and 9 genera. Out of these, Diptera was most diversified and consists of major floral visitors

\section{Keywords}

Diversity, Diptera,

Episyrphus, Apis

florea, Pollination

Article Info

Accepted:

08 July 2018

Available Online:

10 August 2018 followed by Hymenoptera, Coleoptera, Hemiptera and Lepidoptera. Irrespective of different day hours, significantly maximum abundance recorded by Episyrphus sp. followed by Sphaerophora sp. and A. florea. Peak abundance of Episyrphus sp. and A. florea were recorded between $1000 \mathrm{~h}-1200 \mathrm{~h}$ during full bloom of crop. Minimum abundance was recorded at $0600 \mathrm{~h}-0800 \mathrm{~h}$ irrespective of weeks. Present research findings helpful in identification of insects which are helpful in pollination. Farmers will be saved from misconception of every insect is a pest and helpful in reducing pesticide use and maintaining pollinators diversity and also provided lead for future research for exploitation of underutilized fruit crops. Integration of indigenous underutilized fruits could be one of the appropriate facets to enhance overall horticultural production in the region. Being tolerant to biotic and abiotic stresses, these fruit species are suitable for growing in the disaster- and drought-prone areas. Diversification of agriculture by utilizing these underutilized fruits is a key to doubling farmer's income by providing extra income as their availability is low.

\section{Introduction}

Potential (Underutilized) plant species are only different from other crops because these crops needs to be acknowledged employed and explored for todays' and future generations. A large number of underutilized edible fruits exist in tropic and subtropics of the world. They have been used in Indian system of medicine such as Ayurvedic and Unani since time immemorial. Pollination research based on several fruit crops revealed that management of pollinators had a significant effect in improvement of the qualitative as well as quantitative parameters of all cultivars. An effective pollinator makes sequential visits to the flowers, carries pollen and transfers them to stigma during a visit (Corbet et al., 1991). The utilisation of pollinators especially honeybees is considered as one of the cheapest eco-friendly approaches in maximising the yield of the cross-pollinated 
crops. Lot of work has been done in India and abroad on commercial fruit crops as bee forage but scanty information is available on underutilized fruit and medicinal plants such as Phyllanthus emblica L. (Aonla).

Aonla (Phyllanthus emblica L.), also known as Indian gooseberry, Amla or Emblic, belongs to the family Euphorbiaceae and is native of tropical south eastern Asia. It is grown commercially in India particularly in Uttar Pradesh (FAO, 1982). Fruits are generally used in cooking, preserves, sauce, pickles, jams and jellies, etc. The fruits are very rich in vitamin C (500-600 mg/100g pulp) and have great importance in Ayurvedic medicine (Ghosal et al., 1996) having antiscorbutic properties and used in treatment of ailments associated with digestive system. Aonla is a highly branched monoecious shrub or tree growing up to 9-18 $\mathrm{m}$ high. Leaves are linear-oblong, obtuse, nearly sessile distichously branched on slender branchlets. Small greenish yellow flowers are borne in compact clusters in the axils of the lower leaves. The male flowers are present usually at the lower end of branchlets with female flowers above them. Occasionally, the trees are dioecious. Fruit is a hard berry, round, indented at base and smooth obscurely 6 lobed. The stone contains 6 small seeds (Brun et al., 1987). Present studies were conducted with the objectives to know about species richness and abundance of insect visitors of aonla.

\section{Materials and Methods}

Species richness of insect visitors/pollinators of aonla

Present studies on aonla (Phyllanthus emblica L.) were conducted during 2014 and 2015 at Research Farm of the Department of Horticulture, CCS Haryana Agricultural University, Hisar, India. To record the species richness of insect visitors and pollinators of Phyllanthus emblica L. were collected by sweep net during their blooming period i.e., March -April 2014 and 2015. Sweeps were made at two hourly intervals from the morning to the evening throughout flowering period of the crop (0600h-1600h).

\section{Abundance of insect visitors/pollinators of aonla}

Abundance of different insect visitors/ pollinators, number of visitors $/ \mathrm{m}^{2}$ branch of a tree/5minutes was recorded from five randomly selected branches at two hourly intervals, starting from commencement to the cessation of insect activity and repeated at weekly intervals during blooming period.

Recorded data were analyzed in Randomized Block Design (Snedecor and Cochran, 1989) and the results were compared.

\section{Results and Discussion}

Species richness of insect visitors/pollinators of aonla

Aonla blossoms attracted insects belonging to 5 orders, 7 families, 9 genera and 12 species. Of all these insects, four belongs to order Hymenoptera, two to Coleoptera, four to Diptera, one to Hemiptera and one to Lepidoptera. Among these, Dipterans were the major floral visitors comprising of three families viz., Syrphidae (Sphaerophoria sp. and Episyrphus sp.), Sarcophagidae (Sarcophaga sp.) and Muscidae (Musca domestica). They were followed in order of diversity by hymenopterans from one family viz., Apidae (Apis florea, A. dorsata, A. cerana and A. mellifera). Coleopterans belong to family, Coccinellidae (Coccinella septempunctata and Chilomenes sexmaculata), Hemipteran to Scutellaridae (Chrysocoris stolli) and one Lepidopteran to Arctiidae 
(Amata sp.). Out of 12 insects, all were top foragers except Amata sp. which was side forager also.

\section{Abundance of major insect visitors/pollinators of aonla}

During 2014, three species of flower visiting insects belonging to two orders, namely Hymenoptera (1), and Diptera (2) were collected from the aonla flowers (Table 2). The majority belonged to Diptera comprising syrphid flies (Episyrphus sp. and Sphaerophoria sp.). Among Dipterans, maximum mean population of Episyrphus sp. ( $8.57 \mathrm{flies} / \mathrm{m}^{2}$ branch $/ 5 \mathrm{~min}$ ) followed by that of Sphaerophoria sp. (4.47 flies $/ \mathrm{m}^{2}$ branch $/ 5$ min) (Table 2). In Hymenoptera, Apis florea was recorded with mean population of 3.53 bees $/ \mathrm{m}^{2}$ branch $/ 5 \mathrm{~min}$. Similar trend followed during 2015, Among Dipterans, maximum mean population of Episyrphus sp. (8.56 flies $/ \mathrm{m}^{2}$ branch $/ 5 \mathrm{~min}$ ) followed by that of Sphaerophoria sp. (3.54 flies $/ \mathrm{m}^{2}$ branch/5 min) (Table 3). In Hymenoptera, mean population of Apis florea was 5.70 bees $/ \mathrm{m}^{2}$ branch $/ 5 \mathrm{~min}$.

Present pollination study revealed that Dipteran insect species were most abundant insect pollinators on aonla flowers. Irrespective of different day hours, significantly maximum number of Episyrphus sp. was recorded from aonla flowers followed by Sphaerophora $s p$. and A. florea. Peak abundance of Episyrphus sp. was recorded between 1000h-1200h during full bloom of crop. Maximum abundance of A. florea was recorded at $1000 \mathrm{~h}-1200 \mathrm{~h}$ and minimum abundance was recorded at $0600 \mathrm{~h}-0800 \mathrm{~h}$ irrespective of weeks. During 2014 and 2015, highest pooled mean of abundance ( 8.79 and $8.56 \mathrm{flies} / \mathrm{m}^{2}$ branch $/ 5 \mathrm{~min}$ ) was recorded in Episyrphus sp. followed by A. florea (5.40 and 5.70 bees $/ \mathrm{m}^{2}$ branch $/ 5 \mathrm{~min}$ ), while lowest pooled mean of abundance was recorded in Sphaerophoria sp. (3.62 and 3.54 flies $/ \mathrm{m}^{2}$ branch/5min) (Table 1-3).

Table.1 Diversity of Insect visitors/pollinators of Aonla

\begin{tabular}{|c|c|c|c|c|}
\hline Order & Family & Insect Species & IP/IV* & Working Behaviour \\
\hline \multirow[t]{4}{*}{ Hymenoptera } & \multirow[t]{4}{*}{ Apidae } & Apis florea Fabricius & IP & $\mathrm{T}$ \\
\hline & & Apis dorsata Fabricius & IP & $\mathrm{T}$ \\
\hline & & Apis cerana Fabricius & IP & $\mathrm{T}$ \\
\hline & & Apis mellifera Linnaeus & IP & $\mathrm{T}$ \\
\hline \multirow[t]{2}{*}{ Coleoptera } & \multirow[t]{2}{*}{ Coccinellidae } & $\begin{array}{l}\text { Coccinella septempunctata } \\
\text { Linnaeus }\end{array}$ & IV & $\mathrm{T}$ \\
\hline & & $\begin{array}{l}\text { Chilomenes sexmaculata } \\
\text { Fabricius }\end{array}$ & IV & $\mathrm{T}$ \\
\hline \multirow[t]{4}{*}{ Diptera } & \multirow[t]{2}{*}{ Syrphidae } & Sphaerophoria sp. & IP & $\mathrm{T}$ \\
\hline & & Episyrphus sp. & IP & $\mathrm{T}$ \\
\hline & Sarcophagidae & Sarcophaga sp. & IV & $\mathrm{T}$ \\
\hline & Muscidae & Musca domestica Linnaeus & IV & $\mathrm{T}$ \\
\hline Hemiptera & Scutellaridae & Chrysocoris stolli Wolff & IV & $\mathrm{T}$ \\
\hline Lepidoptera & Arctiidae & Amata sp. & IV & $\mathrm{T}$ and $\mathrm{S}$ \\
\hline
\end{tabular}

*IP -Insect Pollinator and IV - Insect Visitor

*T - Top worker and S- Side worker 
Table.2 Abundance of insect visitors/pollinators on Aonla flowers during 2014

\begin{tabular}{|c|c|c|c|c|c|c|c|c|c|c|c|c|c|c|c|c|c|c|c|c|c|c|c|}
\hline \multirow{3}{*}{$\begin{array}{l}\text { S. } \\
\text { No. }\end{array}$} & \multirow[t]{3}{*}{ Insect visitors } & & & & & & & & & & & & & & & & & & & & & & \\
\hline & & \multicolumn{6}{|c|}{$25 / 3 / 14$} & \multirow{2}{*}{$\begin{array}{c}\text { Over } \\
\text { all } \\
\text { mean }\end{array}$} & \multicolumn{6}{|c|}{$1 / 4 / 14$} & \multirow{2}{*}{$\begin{array}{l}\text { Over } \\
\text { all } \\
\text { mean }\end{array}$} & \multicolumn{6}{|c|}{$8 / 4 / 14$} & \multirow{2}{*}{$\begin{array}{l}\text { Over } \\
\text { all } \\
\text { mean }\end{array}$} & \multirow{2}{*}{$\begin{array}{l}\text { Pooled } \\
\text { mean }\end{array}$} \\
\hline & & $\begin{array}{l}\text { 0600h- } \\
0800 h\end{array}$ & $\begin{array}{l}0800- \\
1000 h\end{array}$ & $\begin{array}{l}1000 \mathrm{~h}- \\
1200 \mathrm{~h}\end{array}$ & $\begin{array}{l}1200 \mathrm{~h}- \\
1400 \mathrm{~h}\end{array}$ & $\begin{array}{l}1400 \mathrm{~h}- \\
1600 \mathrm{~h}\end{array}$ & $\begin{array}{l}1600 \mathrm{~h}- \\
1800 \mathrm{~h}\end{array}$ & & $\begin{array}{l}\text { 0600h- } \\
0800 h\end{array}$ & $\begin{array}{l}0800- \\
1000 h\end{array}$ & $\begin{array}{l}1000 \mathrm{~h}- \\
1200 \mathrm{~h}\end{array}$ & $\begin{array}{l}1200 \mathrm{~h}- \\
1400 \mathrm{~h}\end{array}$ & $\begin{array}{l}1400 \mathrm{~h}- \\
1600 \mathrm{~h}\end{array}$ & $\begin{array}{l}1600 \mathrm{~h}- \\
1800 \mathrm{~h}\end{array}$ & & $\begin{array}{l}\text { 0600h- } \\
0800 h\end{array}$ & $\begin{array}{l}0800- \\
1000 h\end{array}$ & $\begin{array}{l}1000 \mathrm{~h}- \\
1200 \mathrm{~h}\end{array}$ & $\begin{array}{l}1200 \mathrm{~h}- \\
1400 \mathrm{~h}\end{array}$ & $\begin{array}{l}1400 \mathrm{~h}- \\
1600 \mathrm{~h}\end{array}$ & $\begin{array}{l}1600 \mathrm{~h}- \\
1800 \mathrm{~h}\end{array}$ & & \\
\hline 1 & Episyrphus sp. & $\begin{array}{l}9.40 \\
(3.22)\end{array}$ & $\begin{array}{l}9.40 \\
(3.21)\end{array}$ & $\begin{array}{l}12.40 \\
(3.65)\end{array}$ & $\begin{array}{l}9.20 \\
(3.19)\end{array}$ & $\begin{array}{l}7.40 \\
(2.89)\end{array}$ & $\begin{array}{c}3.60 \\
(2.14)\end{array}$ & $\begin{array}{l}8.57 \\
(3.05)\end{array}$ & $\begin{array}{l}13.00 \\
(3.74)\end{array}$ & $\begin{array}{l}12.40 \\
(3.65)\end{array}$ & $\begin{array}{l}14.00 \\
(3.87)\end{array}$ & $\begin{array}{l}7.40 \\
(2.89)\end{array}$ & $\begin{array}{l}10.40 \\
(3.37)\end{array}$ & $\begin{array}{l}5.00 \\
(2.44)\end{array}$ & $\begin{array}{l}10.37 \\
(3.33)\end{array}$ & $\begin{array}{l}12.40 \\
(3.65)\end{array}$ & $\begin{array}{c}7.80 \\
(2 . .96)\end{array}$ & $\begin{array}{l}7.80 \\
(2.96)\end{array}$ & $\begin{array}{l}5.80 \\
(2.60)\end{array}$ & $\begin{array}{l}8.40 \\
(3.06)\end{array}$ & $\begin{array}{l}2.40 \\
(1.83)\end{array}$ & $\begin{array}{l}7.43 \\
(2.84)\end{array}$ & $\begin{array}{l}8.79 \\
(3.07)\end{array}$ \\
\hline 2 & Apis florea & $\begin{array}{l}0.00 \\
(1.00)\end{array}$ & $\begin{array}{l}5.00 \\
(2.44)\end{array}$ & $\begin{array}{l}8.80 \\
(3.12)\end{array}$ & $\begin{array}{l}4.20 \\
(2.27)\end{array}$ & $\begin{array}{l}3.20 \\
(2.04)\end{array}$ & $\begin{array}{r}0.00 \\
(1.00)\end{array}$ & $\begin{array}{l}3.53 \\
(1.98)\end{array}$ & $\begin{array}{c}0.40 \\
(1.16)\end{array}$ & $\begin{array}{l}5.20 \\
(2.47)\end{array}$ & $\begin{array}{l}12.80 \\
(3.71)\end{array}$ & $\begin{array}{l}10.20 \\
(3.34)\end{array}$ & $\begin{array}{r}4.60 \\
(2.36)\end{array}$ & $\begin{array}{l}0.40 \\
(1.14)\end{array}$ & $\begin{array}{l}5.60 \\
(2.36)\end{array}$ & $\begin{array}{l}0.00 \\
(1.00)\end{array}$ & $\begin{array}{l}9.20 \\
(3.18)\end{array}$ & $\begin{array}{l}13.80 \\
(3.84)\end{array}$ & $\begin{array}{l}12.00 \\
(3.60)\end{array}$ & $\begin{array}{l}7.20 \\
(2.86)\end{array}$ & $\begin{array}{l}0.20 \\
(1.08)\end{array}$ & $\begin{array}{l}7.07 \\
(2.59)\end{array}$ & $\begin{array}{l}5.40 \\
(2.31)\end{array}$ \\
\hline 3 & $\begin{array}{l}\text { Sphaerophoria } \\
\text { sp. }\end{array}$ & $\begin{array}{l}7.60 \\
(2.93)\end{array}$ & $\begin{array}{l}3.80 \\
(2.18)\end{array}$ & $\begin{array}{l}5.80 \\
(2.60)\end{array}$ & $\begin{array}{l}3.20 \\
(2.04)\end{array}$ & $\begin{array}{l}4.20 \\
(2.27)\end{array}$ & $\begin{array}{r}2.20 \\
(1.77)\end{array}$ & $\begin{array}{l}4.47 \\
(2.30)\end{array}$ & $\begin{array}{l}4.20 \\
(2.27)\end{array}$ & $\begin{array}{l}4.80 \\
(2.40)\end{array}$ & $\begin{array}{l}7.00 \\
(2.82)\end{array}$ & $\begin{array}{r}5.00 \\
(2.43)\end{array}$ & $\begin{array}{c}3.20 \\
(2.04)\end{array}$ & $\begin{array}{c}1.80 \\
(1.65)\end{array}$ & $\begin{array}{l}4.33 \\
(2.27)\end{array}$ & $\begin{array}{r}1.80 \\
(1.65)\end{array}$ & $\begin{array}{l}4.40 \\
(2.31)\end{array}$ & $\begin{array}{c}3.00 \\
(1.99)\end{array}$ & $\begin{array}{c}1.60 \\
(1.59)\end{array}$ & $\begin{array}{c}1.00 \\
(1.39)\end{array}$ & $\begin{array}{c}0.60 \\
(1.24)\end{array}$ & $\begin{array}{c}2.07 \\
(1.70)\end{array}$ & $\begin{array}{l}3.62 \\
(2.09)\end{array}$ \\
\hline & Mean & $\begin{array}{c}5.67 \\
(2.38)\end{array}$ & $\begin{array}{c}6.07 \\
(2.61)\end{array}$ & $\begin{array}{l}9.00 \\
(3.12)\end{array}$ & $\begin{array}{c}5.53 \\
(2.50)\end{array}$ & $\begin{array}{l}4.93 \\
(2.40)\end{array}$ & $\begin{array}{c}1.93 \\
(1.63)\end{array}$ & $\begin{array}{c}5.52 \\
(2.44)\end{array}$ & $\begin{array}{c}5.87 \\
(2.39)\end{array}$ & $\begin{array}{l}7.47 \\
(2.84)\end{array}$ & $\begin{array}{l}11.27 \\
(3.46)\end{array}$ & $\begin{array}{l}7.53 \\
(2.89)\end{array}$ & $\begin{array}{c}6.07 \\
(2.59)\end{array}$ & $\begin{array}{c}2.40 \\
\text { (1.74) }\end{array}$ & $\begin{array}{l}6.77 \\
(2.65)\end{array}$ & $\begin{array}{l}4.73 \\
(2.10)\end{array}$ & $\begin{array}{c}7.13 \\
(2.82)\end{array}$ & $\begin{array}{c}8.20 \\
(2.93)\end{array}$ & $\begin{array}{l}6.47 \\
(2.60)\end{array}$ & $\begin{array}{c}5.53 \\
(2.44)\end{array}$ & $\begin{array}{c}1.07 \\
(1.39)\end{array}$ & $\begin{array}{l}5.52 \\
(2.38)\end{array}$ & $\begin{array}{c}5.94 \\
(2.49)\end{array}$ \\
\hline
\end{tabular}

Each value represents mean of 5 observations

*Figures in parentheses are square root transformed values

\begin{tabular}{|l|l|l|}
\hline Factor & SEm $( \pm)$ & C.D. \\
\hline Insect visitors & $\mathbf{0 . 0 2}$ & $\mathbf{0 . 0 5}$ \\
\hline Week & $\mathbf{0 . 0 2}$ & $\mathbf{0 . 0 5}$ \\
\hline Time & $\mathbf{0 . 0 2}$ & $\mathbf{0 . 0 7}$ \\
\hline Insect X Week & $\mathbf{0 . 0 3}$ & $\mathbf{0 . 0 9}$ \\
\hline Insect visitors X Time & $\mathbf{0 . 0 4}$ & $\mathbf{0 . 1 3}$ \\
\hline Week X Time & $\mathbf{0 . 0 4}$ & $\mathbf{0 . 1 3}$ \\
\hline Insect visitors X Week Time & $\mathbf{0 . 0 8}$ & $\mathbf{0 . 2 3}$ \\
\hline
\end{tabular}


Table.3 Abundance of insect visitors/pollinators on Aonla flowers during 2015

\begin{tabular}{|c|c|c|c|c|c|c|c|c|c|c|c|c|c|c|c|c|c|c|c|c|c|c|c|}
\hline \multirow{3}{*}{$\begin{array}{l}\text { S. } \\
\text { No. }\end{array}$} & \multirow[t]{3}{*}{ Insect visitors } & \multicolumn{22}{|c|}{ Number of insect visitors $/ \mathrm{m}^{2}$ branch of a tree $/ 5$ minutes } \\
\hline & & \multicolumn{6}{|c|}{$27 / 3 / 15$} & \multirow{2}{*}{$\begin{array}{c}\text { Overall } \\
\text { mean }\end{array}$} & \multicolumn{6}{|c|}{$3 / 4 / 15$} & \multicolumn{2}{|c|}{ Overall mean } & \multicolumn{5}{|c|}{$11 / 4 / 15$} & \multirow{2}{*}{$\begin{array}{c}\text { Overall } \\
\text { mean }\end{array}$} & \multirow{2}{*}{$\begin{array}{l}\text { Pooled } \\
\text { mean }\end{array}$} \\
\hline & & $\begin{array}{l}\text { 0600h- } \\
0800 h\end{array}$ & $\begin{array}{l}0800- \\
1000 h\end{array}$ & $\begin{array}{l}1000 \mathrm{~h}- \\
1200 \mathrm{~h}\end{array}$ & $\begin{array}{l}1200 \mathrm{~h}- \\
1400 \mathrm{~h}\end{array}$ & $\begin{array}{l}1400 \mathrm{~h}- \\
1600 \mathrm{~h}\end{array}$ & $\begin{array}{l}1600 \mathrm{~h}- \\
1800 \mathrm{~h}\end{array}$ & & $\begin{array}{l}\text { 0600h- } \\
\text { 0800h }\end{array}$ & $\begin{array}{l}0800- \\
1000 h\end{array}$ & $\begin{array}{l}1000 \mathrm{~h}- \\
1200 \mathrm{~h}\end{array}$ & $\begin{array}{l}1200 \mathrm{~h}- \\
1400 \mathrm{~h}\end{array}$ & $\begin{array}{l}1400 \mathrm{~h}- \\
1600 \mathrm{~h}\end{array}$ & $\begin{array}{l}1600 \mathrm{~h}- \\
1800 \mathrm{~h}\end{array}$ & & $\begin{array}{c}\text { 0600h- } \\
\text { 0800h }\end{array}$ & $\begin{array}{l}0800- \\
1000 h\end{array}$ & $\begin{array}{l}1000 \mathrm{~h}- \\
1200 \mathrm{~h}\end{array}$ & $\begin{array}{l}1200 \mathrm{~h}- \\
1400 \mathrm{~h}\end{array}$ & $\begin{array}{l}1400 \mathrm{~h}- \\
1600 \mathrm{~h}\end{array}$ & $\begin{array}{l}1600 \mathrm{~h}- \\
1800 \mathrm{~h}\end{array}$ & & \\
\hline 1 & Episyrphus sp. & $\begin{array}{l}10.80 \\
(3.43)\end{array}$ & $\begin{array}{c}4.40 \\
(2.31)\end{array}$ & $\begin{array}{l}12.40 \\
(3.65)\end{array}$ & $\begin{array}{l}7.00 \\
(2.82)\end{array}$ & $\begin{array}{c}8.00 \\
(2.99)\end{array}$ & $\begin{array}{c}3.80 \\
(2.18)\end{array}$ & $\begin{array}{c}7.73 \\
(2.90)\end{array}$ & $\begin{array}{c}8.00 \\
(2.99)\end{array}$ & $\begin{array}{l}13.40 \\
(3.79)\end{array}$ & $\begin{array}{l}14.80 \\
(3.97)\end{array}$ & $\begin{array}{c}7.80 \\
(2.96)\end{array}$ & $\begin{array}{c}9.00 \\
(3.16)\end{array}$ & $\begin{array}{c}4.80 \\
(2.40)\end{array}$ & $\begin{array}{l}9.63 \\
(3.21)\end{array}$ & $\begin{array}{l}13.00 \\
(3.73)\end{array}$ & $\begin{array}{c}9.20 \\
(3.19)\end{array}$ & $\begin{array}{l}9.00 \\
(3.15)\end{array}$ & $\begin{array}{c}6.00 \\
(2.64)\end{array}$ & $\begin{array}{c}9.60 \\
(3.25)\end{array}$ & $\begin{array}{c}3.20 \\
(2.04)\end{array}$ & $\begin{array}{c}8.33 \\
(3.00)\end{array}$ & $\begin{array}{c}8.56 \\
(3.03)\end{array}$ \\
\hline 2 & Apis florea & $\begin{array}{c}0.00 \\
(1.00)\end{array}$ & $\begin{array}{c}8.00 \\
(2.99)\end{array}$ & $\begin{array}{c}6.80 \\
(2.79)\end{array}$ & $\begin{array}{c}2.00 \\
(1.71)\end{array}$ & $\begin{array}{c}2.60 \\
(1.89)\end{array}$ & $\begin{array}{c}0.20 \\
(1.08)\end{array}$ & $\begin{array}{c}3.26 \\
(1.91)\end{array}$ & $\begin{array}{c}0.40 \\
(1.16)\end{array}$ & $\begin{array}{c}6.20 \\
(2.68)\end{array}$ & $\begin{array}{l}12.80 \\
(3.71)\end{array}$ & $\begin{array}{l}11.20 \\
(3.48)\end{array}$ & $\begin{array}{c}4.00 \\
(2.22)\end{array}$ & $\begin{array}{c}0.40 \\
(1.16)\end{array}$ & $\begin{array}{c}5.83 \\
(2.40)\end{array}$ & $\begin{array}{c}0.20 \\
(1.08)\end{array}$ & $\begin{array}{l}11.80 \\
(3.57)\end{array}$ & $\begin{array}{l}14.80 \\
(3.97)\end{array}$ & $\begin{array}{l}13.20 \\
(3.76)\end{array}$ & $\begin{array}{c}8.00 \\
(2.99)\end{array}$ & $\begin{array}{c}0.20 \\
(1.08)\end{array}$ & $\begin{array}{c}8.03 \\
(2.74)\end{array}$ & $\begin{array}{c}5.70 \\
(2.35)\end{array}$ \\
\hline 3 & $\begin{array}{l}\text { Sphaerophoria } \\
\text { sp. }\end{array}$ & $\begin{array}{r}9.80 \\
(3.28)\end{array}$ & $\begin{array}{l}4.60 \\
(2.35)\end{array}$ & $\begin{array}{l}5.20 \\
(2.48)\end{array}$ & $\begin{array}{l}2.60 \\
(1.89)\end{array}$ & $\begin{array}{l}3.20 \\
(2.04)\end{array}$ & $\begin{array}{l}1.60 \\
(1.60)\end{array}$ & $\begin{array}{r}4.50 \\
(2.27)\end{array}$ & $\begin{array}{l}1.40 \\
(1.54)\end{array}$ & $\begin{array}{r}4.40 \\
(2.31)\end{array}$ & $\begin{array}{c}7.20 \\
(2.86)\end{array}$ & $\begin{array}{l}4.00 \\
(2.23)\end{array}$ & $\begin{array}{r}4.20 \\
(2.27)\end{array}$ & $\begin{array}{r}2.60 \\
(1.89)\end{array}$ & $\begin{array}{l}3.96 \\
(2.18)\end{array}$ & $\begin{array}{r}1.40 \\
(1.54)\end{array}$ & $\begin{array}{l}4.20 \\
(2.27)\end{array}$ & $\begin{array}{l}3.80 \\
(2.18)\end{array}$ & $\begin{array}{l}2.20 \\
(1.77)\end{array}$ & $\begin{array}{r}1.00 \\
(1.39)\end{array}$ & $\begin{array}{l}0.40 \\
(1.16)\end{array}$ & $\begin{array}{l}2.16 \\
(1.72)\end{array}$ & $\begin{array}{l}3.54 \\
(2.06)\end{array}$ \\
\hline & Mean & $\begin{array}{l}6.86 \\
(2.38)\end{array}$ & $\begin{array}{r}5.66 \\
(2.61)\end{array}$ & $\begin{array}{l}8.13 \\
(3.12)\end{array}$ & $\begin{array}{l}3.86 \\
(2.50)\end{array}$ & $\begin{array}{l}4.60 \\
(2.40)\end{array}$ & $\begin{array}{r}1.86 \\
(1.63)\end{array}$ & $\begin{array}{l}5.16 \\
(2.36)\end{array}$ & $\begin{array}{l}3.26 \\
(2.39)\end{array}$ & $\begin{array}{r}8.00 \\
(2.84)\end{array}$ & $\begin{array}{l}11.60 \\
(3.46)\end{array}$ & $\begin{array}{l}7.66 \\
(2.89)\end{array}$ & $\begin{array}{l}5.73 \\
(2.59)\end{array}$ & $\begin{array}{r}2.60 \\
(1.74)\end{array}$ & $\begin{array}{l}6.47 \\
(2.60)\end{array}$ & $\begin{array}{l}4.86 \\
(2.10)\end{array}$ & $\begin{array}{r}8.40 \\
(2.82)\end{array}$ & $\begin{array}{l}9.20 \\
(2.93)\end{array}$ & $\begin{array}{l}7.13 \\
(2.60)\end{array}$ & $\begin{array}{l}6.20 \\
(2.44)\end{array}$ & $\begin{array}{r}1.26 \\
(1.39)\end{array}$ & $\begin{array}{l}6.17 \\
(2.49)\end{array}$ & $\begin{array}{r}5.93 \\
(2.48)\end{array}$ \\
\hline
\end{tabular}

Each value represents mean of 5 observations

*Figures in parentheses are square root transformed values

\begin{tabular}{|l|c|c|}
\hline Factor & \multicolumn{2}{|c|}{ SEm $( \pm)$} \\
\hline Insect visitors & $\mathbf{0 . 0 2}$ & $\mathbf{0 . 0 5}$ \\
\hline Week & $\mathbf{0 . 0 2}$ & $\mathbf{0 . 0 5}$ \\
\hline Time & $\mathbf{0 . 0 2}$ & $\mathbf{0 . 0 7}$ \\
\hline Insect X Week & $\mathbf{0 . 0 3}$ & $\mathbf{0 . 0 9}$ \\
\hline Insect visitors X Time & $\mathbf{0 . 0 4}$ & $\mathbf{0 . 1 3}$ \\
\hline Week X Time & $\mathbf{0 . 0 4}$ & $\mathbf{0 . 1 3}$ \\
\hline Insect visitors X Week Time & $\mathbf{0 . 0 8}$ \\
\hline
\end{tabular}




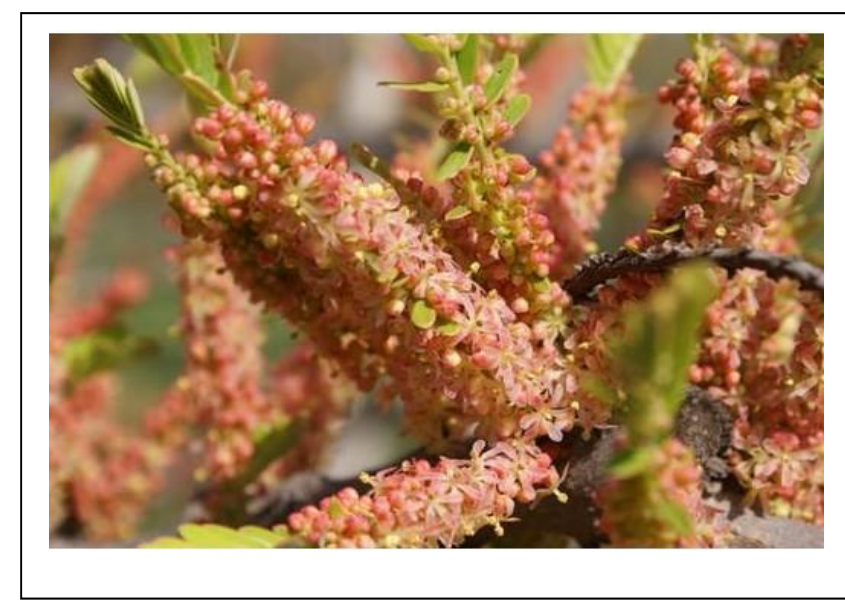

I. BLOSSOMS OF PHYLLANTHUS EMBLICA L.

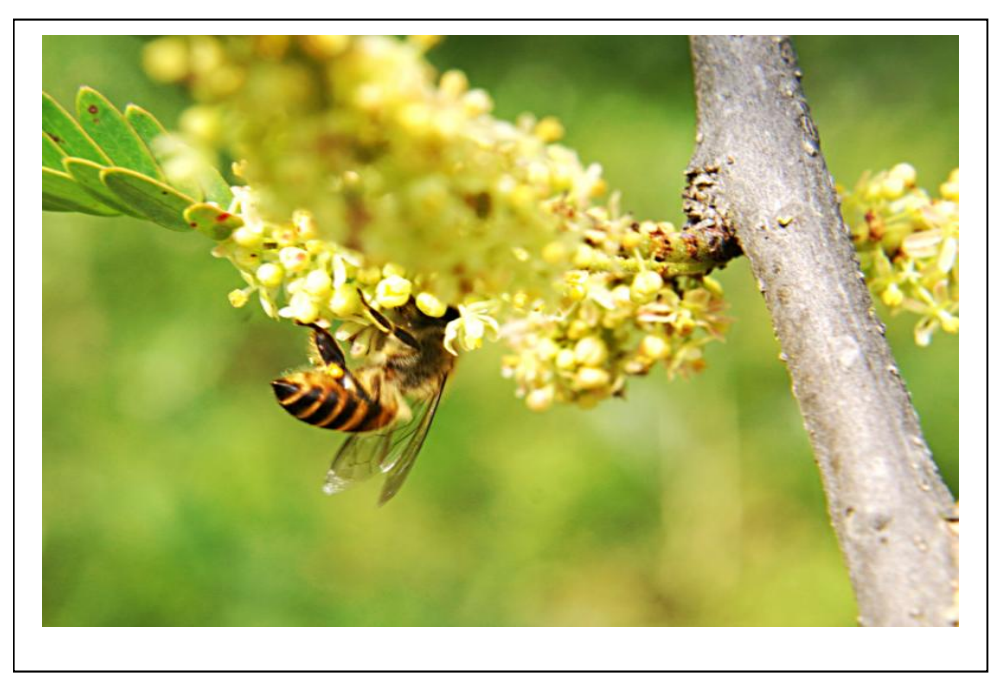

III. APIS MELLIFERA

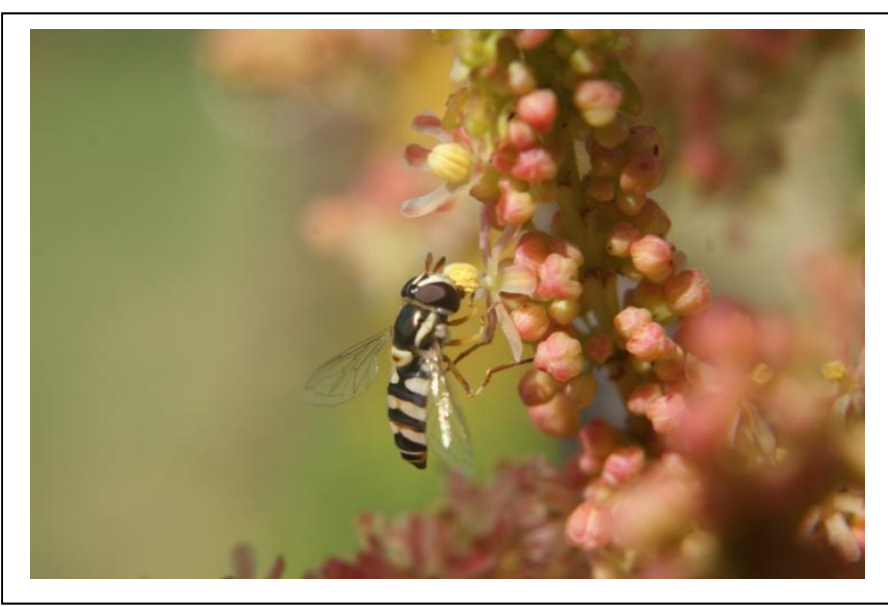

II. SPHAEROPHORIA SP.

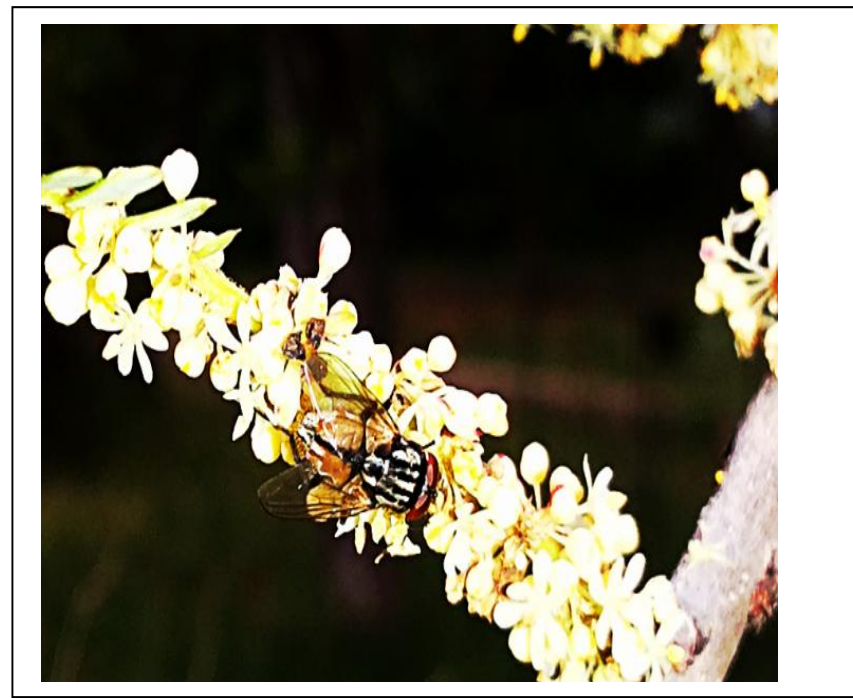

IV. MUSCA SP. 


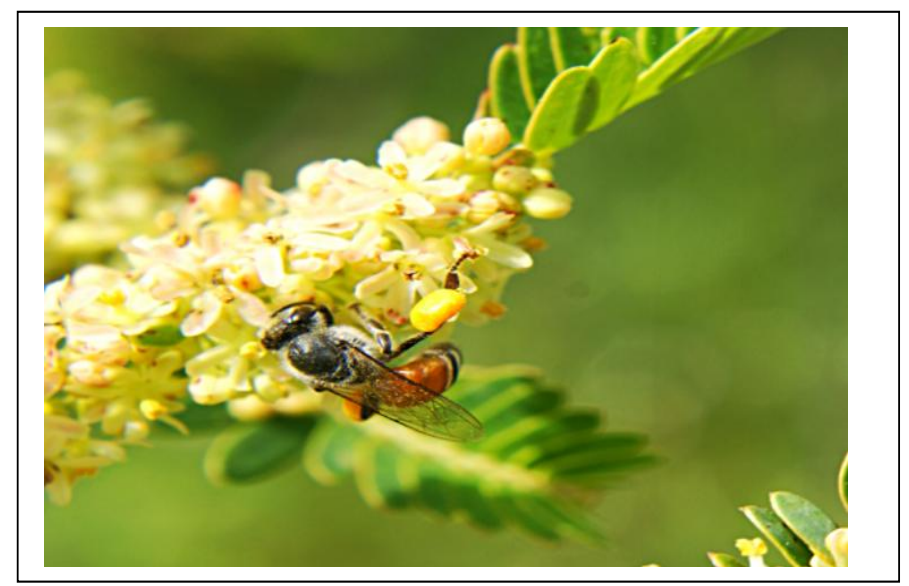

V. APIS FLOREA

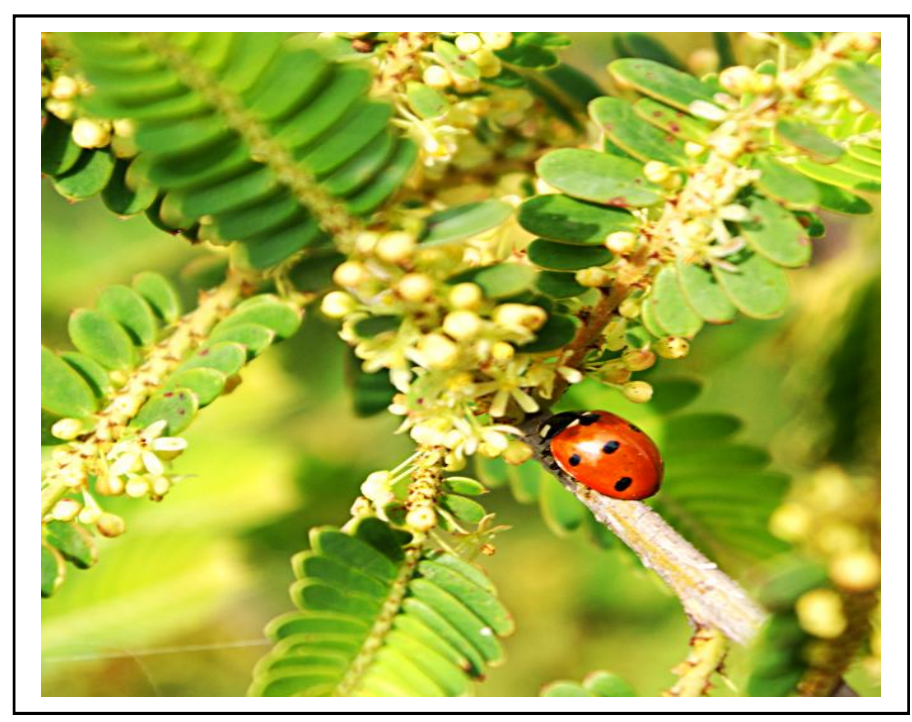

VII. COCCINELLA SEPTEMPUNCTATA

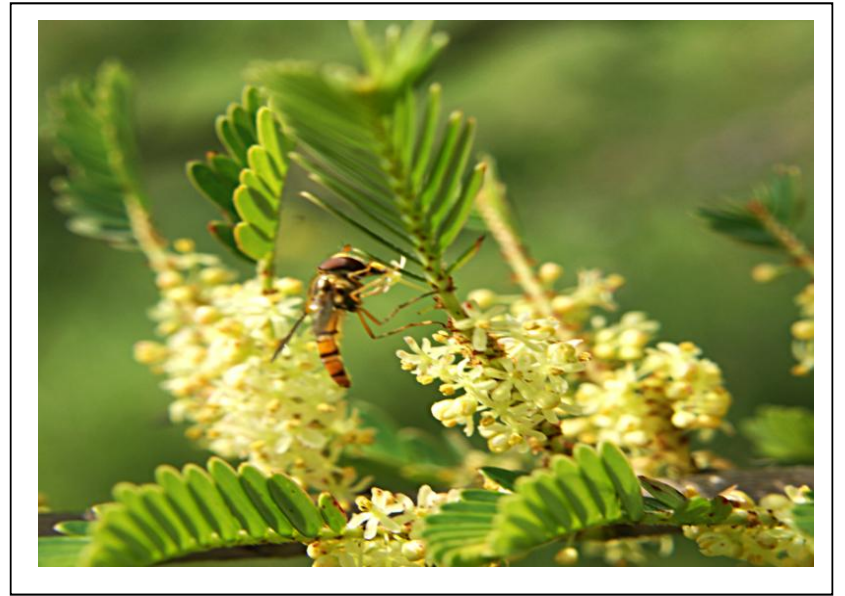

VI. EPISYRPHUS SP.

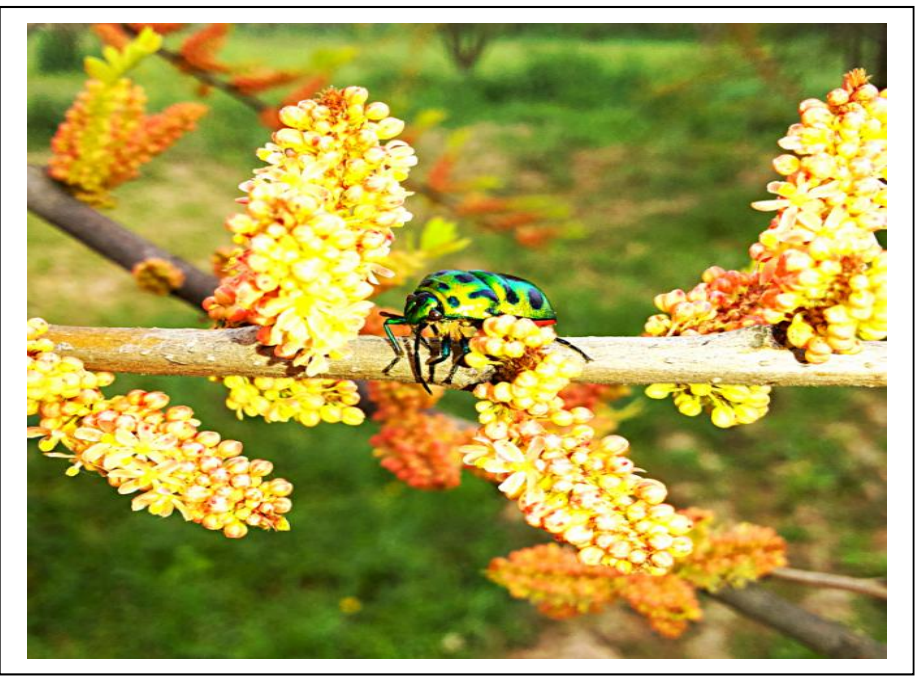

viii. CHRYSOCORIS STOLLI 
The results pertaining to the species richness of insect visitors/pollinators on aonla during March -April 2014 and 2015 revealed that Dipterans were the major floral visitors followed by Hymenoptera, Coleoptera, Hemiptera and Lepidoptera. Similar findings were reported in aonla by Saini (2011) and stated that majority of insect visitors belonged to Diptera followed by Hymenoptera and Lepidoptera. Dipteran insect species were most abundant insect pollinators on aonla flowers. Peak abundance of Episyrphus sp. was recorded between $1000 \mathrm{~h}-1200 \mathrm{~h}$ during full bloom of crop. Maximum abundance of A. florea was recorded at $1000-1200 \mathrm{~h}$ and minimum abundance was recorded at $0600 \mathrm{~h}$ 0800h irrespective of weeks. Irrespective of different day hours, significantly maximum number of Episyrphus sp. was recorded from aonla flowers followed by Sphaerophora $s p$. and A. florea. Present findings are contradictary with the studies reported by Saini (2011) found that hymenopterans are mose abundant followed by dipterans in amla.

\section{References}

Brun, V., and Schumacher, T. 1987. Traditional Herbal Medicine in
Northern Thailand. University of California Press, Berkeley, pp. 349.

Corbet, S. A., I. H. Williams and Osborne, J. L. 1991. Bees and the pollination of crops and wild flowers in the European community. Bee World, 72(2): 47-59.

FAO 1982. Fruit bearing forest trees. FAO Forestry paper No. 34. FAO, Rome, pp. 177.

Ghosal, S., V. K. Tripathi and Chauhan, S. 1996. Active constituent of Emblica officinalis: part 1 st the chemistry and antioxidant effects of two new hydrolysable tannins, emblicanin A and B. Indian Journal of Chemistry, 35b: 941-948.

Saini, R. 2011. Studies on the pollination mechanism in different varieties of amla (Emblica officinalis L.). Ph. D Thesis, CCS Haryana Agricultural University, Hisar, India.

Sihag, R. C. 1986. Insect pollination increases seed production in cruciferous and umbelliferous crops. Journal of Apicultural Research, 25(2): 121-126.

Snedecor, G. W., and Cochran, W. G. 1989. Statistical methods. Oxford and IBH publishing Company, Delhi.

\section{How to cite this article:}

Anoosha Vadde, Sumit Saini, H.D. Kaushik and Sunita Yadav. 2018. Species Richness and Abundance of Major Insect Pollinators on Aonla, (Phyllanthus emblica L.). Int.J.Curr.Microbiol.App.Sci. 7(08): 1256-1263. doi: https://doi.org/10.20546/ijcmas.2018.708.142 